Khalid, A., Thoms, S., Macintyre, D., Thayne, I.G., and Cumming, D.R.S. (2014) Fabrication of submicron planar Gunn diode. In: 26th International Conference on Indium Phosphide and Related Materials, 11-15 May 2014, Montpelier, France.

Copyright (C) 2014 The Authors

A copy can be downloaded for personal non-commercial research or study, without prior permission or charge

Content must not be changed in any way or reproduced in any format or medium without the formal permission of the copyright holder(s)

When referring to this work, full bibliographic details must be given

$\underline{\text { http://eprints.gla.ac.uk/94667 }}$

Deposited on: 10 September 2014

Enlighten - Research publications by members of the University of Glasgow_ http://eprints.gla.ac.uk 


\title{
Fabrication of Submicron Planar Gunn Diode
}

\author{
Ata. Khalid ${ }^{1 *}$, S. Thoms ${ }^{1}$, D. Macintyre ${ }^{1}$, I. G. Thayne ${ }^{1}$ and D.R.S. Cumming ${ }^{1}$ \\ ${ }^{1}$ : School of Engineering, University of Glasgow, Glasgow, United Kingdom, G12 8LT \\ e-mail: ata.khalid@glasgow.ac.uk
}

\begin{abstract}
Short-Abstract-We present, for the first time, the fabrication process for a submicron planar Gunn diode in $\mathrm{In}_{0.53} \mathrm{Ga}_{0.47} \mathrm{As}$ on an InP substrate operating at $265 \mathrm{GHz}$. A novel two stage lift off method has been developed to achieve a submicron gaps between contacts down to $135 \mathrm{~nm}$ with widths up to $120 \mu \mathrm{m}$.
\end{abstract}

\section{INTRODUCTION}

Semiconductor device fabrication technology is capable of very small feature sizes but some III-V devices such as Gunn diodes require dimensions longer than a micron with submicron sized gaps. Writing narrow gaps between long and wide contacts is difficult by electron beam lithography despite the small beam diameter due to electron beam proximity effects. The effective dose within the gap is $50 \%$ of the clearing dose, and with a conventional polymethylmethacrylate (PMMA) lift off process this leads to rapid resist erosion and a process window of around $1 \%$ in dose. Unfortunately, it is relatively difficult to etch high resolution gold patterns, and because of this, small gaps between metal contacts are difficult to fabricate using the conventional subtractive processing flow of metal deposition followed by resist patterning and metal etch. Thoms et al [1] previously showed that $100 \mathrm{~nm}$ gaps between $100 \mu \mathrm{m}$ square metal contacts can be realized using a tri-layer of polydimethylglutarimide (PMGI) silicon nitride and UVIII. The UVIII resist layer is patterned by electron beam lithography. Reactive ion etching (RIE) is used to etch through the silicon nitride layer and a low bias oxygen RIE process is used to etch the sacrificial PMGI layer. The silicon nitride layer is not etched by the plasma and so enables the PMGI layer to be etched back by a controlled amount to give profiles optimal for metal lift off.

In this paper, we demonstrate the application of the sacrificial PMGI layer technique used to produce undercut profiles for metal lift off to the fabrication of submicron planar Gunn diodes using an epitaxial layer of $\mathrm{In}_{0.53} \mathrm{Ga}_{0.47} \mathrm{As}$ on an $\mathrm{InP}$ substrate. We show that $700 \mathrm{~nm}$ long devices oscillate at $264 \mathrm{GHz}$ with a measured rf power of $-10 \mathrm{dBm}$. These planar Gunn devices are excellent candidates for many applications requiring a solid-state $\mathrm{THz}$ source for chip integration..

\section{EXPERIMENTAL}

\section{A. Device Fabrication}

InGaAs layers were grown using molecular beam epitaxy (MBE) on a $600 \mu \mathrm{m}$ thick semi-insulating InP substrate, as shown in Figure 1. The active channel layer was $300 \mathrm{~nm}$ thick with a doping level of $8 \times 10^{16} \mathrm{~cm}^{-3}$, followed by a $200 \mathrm{~nm}$ thick cap layer of $\operatorname{In}_{0.53} \mathrm{Ga}_{0.47} \mathrm{As}$ with a doping level of $2 \times 10^{18} \mathrm{~cm}^{-3}$. The cap layer helps to achieve lower Ohmic contact resistance to the devices. The channel layers are designed to keep the $n L_{a c}$ product of the devices above $10^{12} \mathrm{~cm}^{-2}$, where $n$ is the free carrier density and $L_{a c}$ is the separation distance between the anode and cathode [2].

Substrates were initially spin coated with a $300 \mathrm{~nm}$ thick layer of PMGI SF6 (supplied by Microchem) and baked at $180^{\circ} \mathrm{C}$ for $2 \mathrm{hrs}$. A $50 \mathrm{~nm}$ layer of silicon nitride was then applied using a room temperature PECVD process. A $300 \mathrm{~nm}$ thick layer of UVIII resist (supplied by Chestech LTD) was applied to the substrates from a mixture of 60:40 UVIII to ethyl lactate by weight. Substrates were spin coated at $3000 \mathrm{rpm}$ for $60 \mathrm{~s}$ and baked on a vacuum hot plate at $145^{\circ} \mathrm{C}$ for $60 \mathrm{~s}$. A Vistec VB6 UHR EWF large area electron beam lithography tool was then used to pattern anode and cathode pads with dimension $120 \mu \mathrm{m} \times 80 \mu \mathrm{m}$ and a separation of $700 \mathrm{~nm}$. Pattern proximity correction and fracturing was carried out using Layout Beamer software from GenIsys GMBH. After ebeam exposure substrates were given a post exposure bake at $145^{\circ} \mathrm{C}$ for 60 s on a vacuum hot plate. The UVIII layer was developed for 60 s in CD26 at room temperature and washed in DI water. The parting layer of silicon nitride was then etched for $90 \mathrm{~s}$ in CHF3/O2 50/5 sccm, @150 W, 55mT, using an Oxford Instruments RIE80+ tool.

To create an undercut profile suitable for metal lift off the same RIE tool was used with a low bias oxygen etch process (20W at $10 \mathrm{mT}$ and an oxygen flow rate of $10 \mathrm{sccm}$ for $15 \mathrm{mins}$ ) to etch back the sacrificial PMGI layer by a controlled amount. To ensure the PMGI layer was completely etched down to the cap layer an interferometer was used to monitor the etch process throughout. A Plassys $450 \mathrm{MB}$ ebeam evaporator was used to deposit the following metal films to the patterned substrates $\mathrm{Au}(10 \mathrm{~nm}), \mathrm{Ge}(14 \mathrm{~nm}), \mathrm{Au}(10 \mathrm{~nm}), \mathrm{Ni}(14 \mathrm{~nm})$ and $\mathrm{Au}(80 \mathrm{~nm})$. A two step lift off process was found to give reliable metal lift off. In the first step substrates were soaked in acetone which removed the UVIII layer and the excess metal film. A second soak in SVC-14 was used to remove the PMGI resist and wash away the nitride layer. This step was carried out using ultrasound agitation for 5 seconds to ensure substrates were perfectly clean after the lift-off process. The quality of lifted off metal patterns was good. The fabrication process described was found to be very successful at defining wide anode-cathode structures up to $120 \mu \mathrm{m}$ with $L_{a c}$ as short as $130 \mathrm{~nm}$. Figure 2. a shows a typical submicron structure obtained and this has an anode cathode separation of $130 \mathrm{~nm}$. These results are an improvement on those previously reported for the fabrication of $1.3 \mu \mathrm{m}$ long planar Gunn diodes [3].

The contacts were not annealed and contact resistances of the order of $0.12 \Omega \mathrm{mm}$ were achieved. A mesa was etched using 1:1:10:: $\mathrm{H}_{2} \mathrm{SO}_{4}: \mathrm{H}_{2} \mathrm{O}_{2}: \mathrm{H}_{2} \mathrm{O}$ at an etch rate of $60 \mathrm{~nm} / \mathrm{s}$. A $50 \Omega$ coplanar waveguide (CPW) feed structure was then 
deposited using $800 \mathrm{~nm}$ of gold to form the probe pads for subsequent on-wafer RF and dc measurements. The CPW had a ground-to-signal separation of $15 \mu \mathrm{m}$ and a central signal track width of $50 \mu \mathrm{m}$. Finally, the unwanted $\mathrm{In}_{0.53} \mathrm{Ga}_{0.47} \mathrm{As}$ contact layer between the anode and cathode was carefully etched away using 1:1:8 $\mathrm{H}_{3} \mathrm{PO}_{4}: \mathrm{H}_{2} \mathrm{O}_{2}: \mathrm{H}_{2} \mathrm{O}$ solution.

\section{B. $D C \& R F$ Measurements}

DC characteristics of the devices were measured with a semiconductor device analyzer (Agilent Technologies B1500A) on a semi-automated probe station (Cascade Microtech Summit 12K). Figure 3 shows measured currentvoltage characteristics of fabricated $700 \mathrm{~nm}$ long and $120 \mu \mathrm{m}$ wide planar Gunn diode. Conventional vertical Gunn oscillators are frequency band limited because they are constructed in rectangular waveguide cavities; planar Gunn diodes, on the other hand, are integrated with CPWs that have no cut-off frequency limitation. This configuration allows device oscillation frequency to be measured for the entire band using on wafer probing. Figure 4. shows measured spectrum of a submicron planar Gunn diode oscillating above $265 \mathrm{GHz}$.

\section{DISCUSSION}

The experimental results presented in this work explore the function of Gunn operation at submicron lengths. Simulations using Monte Carlo methods have already shown that Gunn diodes may function with submicron transit regions [4]. Since the transit region length is intrinsically related to the frequency at which the devices operate, the new submicron devices discussed in this paper allow increases in the frequency of operation. As the experimental planar Gunn device have a quite highly doped transit region, thermal generation within such a device may be significant, and one area worth further study would be the transport of heat within such a device.

\section{CONCLUSIONS}

In conclusion, the first submicron $(700 \mathrm{~nm} \times 120 \mu \mathrm{m})$ $\mathrm{In}_{0.53} \mathrm{Ga}_{0.47} \mathrm{As} / \mathrm{InP}$ planar Gunn diode has been fabricated and found to oscillate above $265 \mathrm{GHz}$. The highest observed power was above $-10 \mathrm{dBm}$. The fabrication technique lends itself to device arrays to make very high power sources. The proposed $\mathrm{In}_{0.53} \mathrm{Ga}_{0.47} \mathrm{As} / \mathrm{InP}$ planar Gunn diode with a higher level of output power shows great potential as a solid-state source of terahertz radiation.

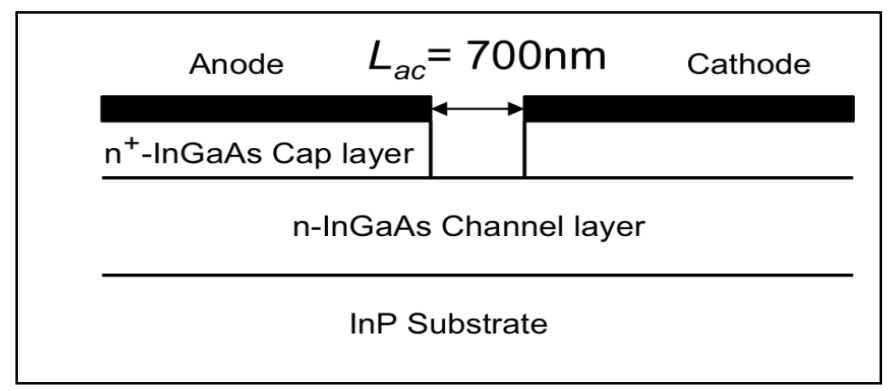

Figure 1. A schematic diagram of the layer structure of the $\operatorname{In}_{0.53} \mathrm{Ga}_{0.47} \mathrm{As}$ device.

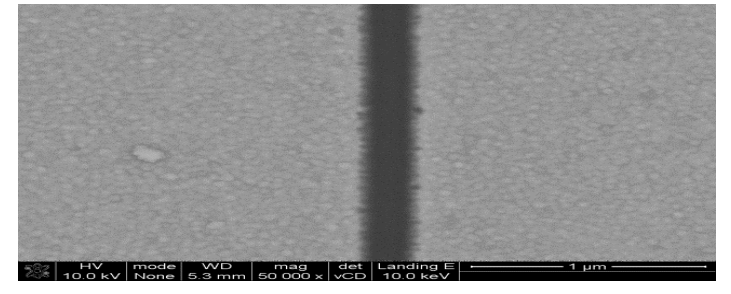

Figure 2. A SEM image taken using FEI scanning electron microscope for a typical submicron planar Gunn diode. The measured gap is $\sim 140 \mathrm{~nm}$.

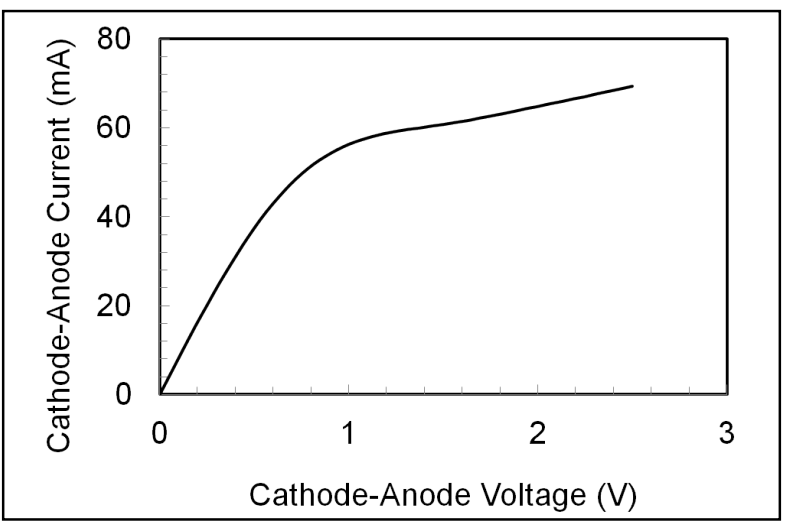

Figure 3. Measured current-voltage characteristics of a completed 700nm x $120 \mu \mathrm{m}$ planar Gunn diode using $500 \mu$ s pulse voltage.

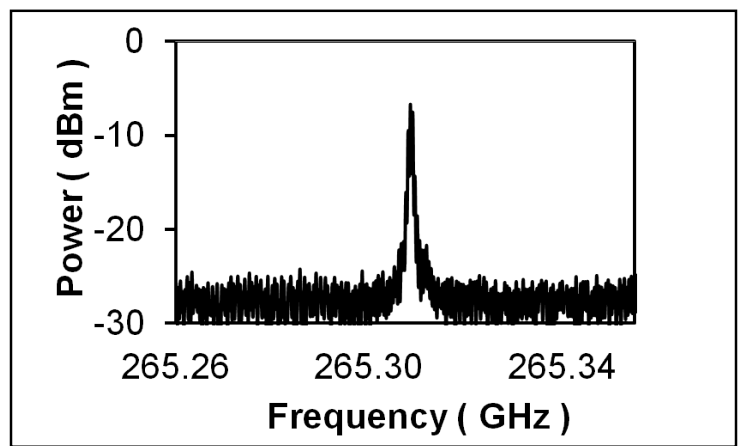

Figure 4. Measured on-wafer spectrum \& power measurement of 700nm planar Gunn diodes using a mixer. The insertion is de-embedded as specified by the manufacturer.

\section{ACKNOWLEDGMENT}

This work was supported by UK EPSRC and e2v Technologies (UK) Ltd.

\section{REFERENCES}

[1] S.Thoms and D.S.Macintyre, "Long nanoscale gaps on III-V substrates by electron beam lithography"J.Vac. Sci. Technol, B 30 06F305 (2012)

[2] W. Kowalsky and A. Schlachetzki, "InGaAs Gunn oscillators," Electron. Lett., vol. 20, no. 12, pp. 502-503, Jun. 1984

[3] A. Khalid, C. Li, V. Papageogiou, G. M. Dunn, M. J. Steer, I. G. Thayne, M. Kuball, C. H. Oxley, M. M. Bajo, A. Stephen, J. Glover, and D. R. S. Cumming, "In0.53Ga0.47As planar Gunn diodes operating at a fundamental frequency of $164 \mathrm{GHz}$ " IEEE Electron Device Lett., vol. 34, no. 1, pp. 39-41, Jan. 2013.

[4] S. Perez, T. González, D. Pardo, and J. Mateos, "Terahertz Gunn-like oscillations in InGaAs/InAlAs planar diode,” J. Appl. Phys., vol.103, no.9, pp.4516-4518, May 2008. 